\title{
MP
}

\section{Influence of suppression of CapG gene expression by siRNA on the growth and metastasis of human prostate cancer cells}

\author{
B.K. Li*, K. Guo*, C.Y. Li, H.L. Li, P.P. Zhao, K. Chen and C.X. Liu \\ Department of Urology, Zhujiang Hospital, Southern Medical University, \\ Guangzhou, Guangdong, China \\ *These authors contributed equally to this study. \\ Corresponding authors: B.K. Li / C.Y. Li \\ E-mail: pk939428@163.com / zhujianglcy@163.com
}

Genet. Mol. Res. 14 (4): 15769-15778 (2015)

Received August 18, 2015

Accepted October 23, 2015

Published December 1, 2015

DOI http://dx.doi.org/10.4238/2015.December.1.28

\begin{abstract}
This study investigated CapG gene expression in prostate cancer cell lines; in addition, we explored the effects of CapG suppression on DU145 cell growth, and the underlying mechanism with which CapG affects DU145 cell growth and invasiveness. The expression of CapG and 18 related genes in DU145 cells was analyzed by flow cytometry, quantitative polymerase chain reaction (qPCR), CCK8 assay, western blot, and the trans-well assay. DU145 cells were transfected with designed small interfering RNA (siRNA). CapG expression was quantified by qPCR and western blot. DU145 cell proliferation and invasiveness was analyzed using the CCK8, flow cytometric, and trans-well assays. CapG, TMPRSS1, EGFR, ETS-1, ERBB2, AKT, Cyclin D1, P21, Bcl-2, and Bak1 gene and Bcl-2, Cyclin D1, and CapG protein expressions were significantly lower in the siRNA group compared to the negative control group $(P<0.05)$. The proliferation of CapG siRNA DU145 cells was lower than that of the two control groups, $48 \mathrm{~h}$ after transfection. The cell inhibition rate was $24.5,35.4$, and $16,5 \%$ at 24,48 , and $72 \mathrm{~h}$, respectively. The growth curve indicated that CapG siRNA DU145 cells showed a significantly slower
\end{abstract}


proliferation rate $(P<0.05)$. The trans-well assay showed a significant decrease in the migratory and invasive capacities of DU145 cells in the siRNA group $(P<0.05)$. The suppression of CapG expression caused a significant decrease in the proliferation, invasiveness, and metastasis of DU145 cells. The mechanism with which CapG, with other oncogenes, influences cancer cell cycle remains to be elucidated.

Key words: Prostate cancer; CapG; RNA interference; Cell growth; Invasion; Metastasis

\section{INTRODUCTION}

Prostate cancer (PCa) is the most common type of cancer affecting men; it is also the second leading cause of cancer deaths in men in the United States, with an estimated 238,590 newly diagnosed cases and 29,720 deaths in 2012 (Siegel et al., 2013). There has been a major increase in the incidence of prostate cancer in China over the past few years, with prostate cancer becoming a major cause of cancer-related deaths in Chinese men. Androgen is an important hormone affecting the progression of $\mathrm{PCa}$; therefore, the androgen receptor (AR) signaling pathway plays a critical role in the progression of PCa (Karantanos et al., 2013). However, the AR signaling pathway is not a single pathway, but a complex signaling network that includes multiple coactivators (Rosenfeld et al., 2006). CapG is a protein with the characteristics of a coactivator. The high expression of CapG has been related to a variety of tumors, and has been reported to promote the metastasis and invasion of tumor cells. CapG is overexpressed in lung cancer, breast cancer, pancreatic cancer, bile duct cancer, and colorectal cancer cells, as well as ovarian tumors and hepatocellular carcinoma. In addition, CapG can be utilized clinically as a part of the treatment for these malignancies (Thompson et al., 2007; Partheen et al., 2008; Zheng et al., 2010; Shao et al., 2011; Wu et al., 2011; Morofuji et al., 2012; Zhu et al., 2012; Kimura et al., 2013). Therefore, CapG has certain clinical prospects in the treatment and diagnosis of various types of cancer.

In this study, we investigated the expression of CapG in prostate cancer. We found that the downregulation of CapG inhibited cell proliferation and metastasis. Our results suggested that CapG may be associated with aggressive prostate cancer.

\section{MATERIAL AND METHODS}

\section{Cell culture}

Human prostate cancer cell lines (PC3, LNCaP, and DU145) were obtained from the Guangzhou Institute of Biomedicine and Health, Chinese Academy of Sciences. The PC3, LNCaP, and DU145 cell lines were cultured in RPMI-1640 (Hyclone, Logan, UT, USA) supplemented with $10 \%$ fetal bovine serum (FBS; Hyclone) at $37^{\circ} \mathrm{C}$, under a humidified atmosphere with $5 \% \mathrm{CO}_{2}$.

\section{Real-time quantitative polymerase chain reaction (PCR)}

Total RNA was isolated using TRIzol reagent (Invitrogen, Grand Island, NY, USA), and 
the CDNA was reverse transcribed by RT-PCR. The real-time quantitative PCR amplification system was composed of the Bestar ${ }^{\otimes}$ SybrGreen qPCR MasterMix $(10 \mu \mathrm{L}), 10 \mu \mathrm{M}$ PCR forward primer $(0.5 \mu \mathrm{L}), 10 \mu \mathrm{M}$ PCR reverse primer $(0.5 \mu \mathrm{L})$, cDNA $(1 \mu \mathrm{L})$, and $\mathrm{ddH}_{2} \mathrm{O}(8 \mu \mathrm{L})$, for a total volume of $20 \mu \mathrm{L}$. The reaction mix was then prepared according to the manufacturer protocols. The reaction conditions were set as follows: initial denaturation at $94^{\circ} \mathrm{C}$ for $2 \mathrm{~min}$, and 40 cycles of denaturation at $94^{\circ} \mathrm{C}$ for $20 \mathrm{~s}$, annealing at $58^{\circ} \mathrm{C}$ for $20 \mathrm{~s}$, and extension at $72^{\circ} \mathrm{C}$ for $20 \mathrm{~s}$. Realtime PCR was performed using the SYBR Green PCR Master Mix manufactured by PE Applied Biosystems (Warrington, UK), with an ABI Prism 7500 Sequence Detection System (Applied Biosystems, Carlsbad, CA, USA). The comparative Ct method ( $\left.2^{-\Delta \Delta C t}\right)$ was adopted to evaluate the relative quantitation. Each real-time analysis was performed in triplicate. The primers used for the amplification are listed in Table 1. The screening of CapG in the PC3, LNCaP, and Du145 cell lines revealed a high percentage of CapG mRNA in the Du145 cell lines $(P<0.05$; Figure 1). Therefore, the Du145 cells were selected for all future experiments.

Table 1. Primers for real-time quantitative polymerase chain reaction.

\begin{tabular}{|c|c|}
\hline CapG & F: 5'-CAATGAGTCTGACCTCTTC-3' \\
\hline CapG & R: 5'-GTGAAATGCTGACTCCACACCA-3' \\
\hline AR & F: 5'-AGAGCTGCATCAGTTCGCTT-3' \\
\hline AR & R: 5'-AAAGTCCACGCTCACCATGT-3' \\
\hline TMPRSS1 & F: 5'-TGAGGACAGCATCTCTCGGA-3' \\
\hline TMPRSS1 & R: 5'-ACTTTGGTGTAGACGCCTGG-3' \\
\hline ETS-1 & F: 5'-GATAGTTGTGATCGCCTCACC-3' \\
\hline ETS-1 & R: 5'-GTCCTCTGAGTCGAAGCTGTC-3' \\
\hline IGF-1 & F: 5'-GCTCTTCAGTTCGTGTGTGGA-3' \\
\hline IGF-1 & R: 5'-GCCTCCTTAGATCACAGCTCC-3' \\
\hline FGF8 & F: 5'-CAACTCTACAGCCGCACCAGC-3' \\
\hline FGF8 & R: 5'-TGCTCTTGGCGATCAGCTTC-3' \\
\hline EGFR & F: 5'-AGGCACGAGTAACAAGCTCAC-3' \\
\hline EGFR & R: 5'-ATGAGGACATAACCAGCCACC-3' \\
\hline C-FLIP & F: 5'-GTTCAAGGAGCAGGGACAAG-3' \\
\hline C-FLIP & R: 5'-ATCAGGACAATGGGCATAGG-3' \\
\hline E-Cadherin & F: 5'-GGATGTGCTGGATGTGAATG-3' \\
\hline E-Cadherin & R: 5'-CACATCAGACAGGATCAGCAGAA-3' \\
\hline Maspin & F: 5'-ACTGAAACTAATCAAGCGGCTC-3' \\
\hline Maspin & R: 5'-CTTTGCATACGGTCTCTTCGTAG-3' \\
\hline ERBB2 & F: 5'-TGGCCTGTGCCCACTATAAG-3' \\
\hline ERBB2 & R: 5'-AGGAGAGGTCAGGTTTCACAC-3' \\
\hline AKT & F: 5'-GCGACGTGGCTATTGTGAAG-3' \\
\hline AKT & R: 5'-GCAGCGGATGATGAAGGTGT-3' \\
\hline Cyclin D1 & F: 5'-TGGAGCCCGTGAAAAAGAGC-3' \\
\hline Cyclin D1 & R: 5'-TCTCCTTCATCTTAGAGGCCAC-3' \\
\hline P21 & F: 5'-CCAGGCCAAGATGGTGTCTT-3' \\
\hline P21 & R: 5'-TGAGAAAGGATCAGCCATTGC-3' \\
\hline $\mathrm{P} 2$ & F: 5'-AGCGCCAAGGACATCAAGAA-3' \\
\hline P2 & R: 5'-TAACCTTGTTGAGCCGGTCG-3' \\
\hline $\mathrm{Bcl}-2$ & F: 5'-CATGTGTGTGGAGAGCGTCAA-3' \\
\hline $\mathrm{Bcl}-2$ & R: 5'-GCCGGTTCAGGTACTCAGTCA-3' \\
\hline Bak1 & F: 5'-CCCAGGACACAGAGGAGGTTT-3' \\
\hline Bak1 & R: 5'-GCCTCCTGTTCCTGCTGATG-3' \\
\hline FOXO1 & F: 5'-TGATAACTGGAGTACATTTCGCC-3' \\
\hline FOXO1 & R: 5'-CGGTCATAATGGGTGAGAGTCT-3' \\
\hline TRADD & F: 5'-GAGGACTCCACAAACAGGTATG-3' \\
\hline TRADD & R: 5'-GGAGTAGAGCGGAGCCT-3' \\
\hline Cadherin 11 & F: 5'-CCCAGTACACGTTGATGGCT-3' \\
\hline Cadherin 11 & R: 5'-AATGAATTCCGACGGTGGCT-3' \\
\hline GAPDH & F: 5'-TGTTCGTCATGGGTGTGAAC-3' \\
\hline GAPDH & R: 5'-ATGGCATGGACTGTGGTCAT-3' \\
\hline
\end{tabular}




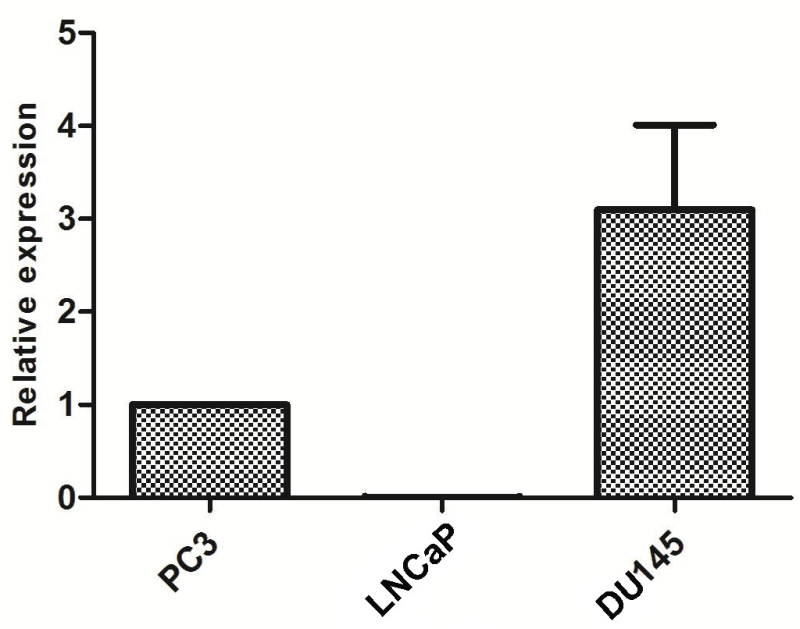

Figure 1. The highest CapG mRNA expression is observed in the DU145 cell line, among the PC3, LNCaP, and DU145 human prostrate cancer cell lines $(P<0.05)$.

\section{Transfection and grouping}

Small interfering RNA (siRNA) were designed and synthesized by Vipotion Biotechnology (Guangzhou, China). The cells were seeded on 24-well plates at a concentration of $3 \times 10^{5}$ per well, and were maintained in RPMI-1640 medium supplemented with $10 \% \mathrm{FBS}$ at $37^{\circ} \mathrm{C}$ and $5 \% \mathrm{CO}_{2}$. Twenty-five nanometer of siRNA was transfected into the cells at a confluence rate of $30-50 \%$ using Lipofectamine RNAiMAX (Invitrogen), according to the manufacturer protocols. The transfection and silencing efficiency were monitored by qPCR. DU145 cells were divided into three groups: the blank control group, the negative control group (transfected with negative control siRNA; siRNA$\mathrm{NC})$, and the experimental transfected group.

\section{Flow cytometry}

The cells were collected after transfection for $48 \mathrm{~h}$, and divided into three groups: the blank control and NC (transfected with negative control siRNA) groups, containing $1 \times 10^{6}$ cells per sample. The cells were harvested, washed twice with ice-cold phosphate-buffered saline (PBS), and fixed overnight with ice-cold $70 \%$ ethanol. The cells were centrifuged at $180 \mathrm{~g}$ for $5 \mathrm{~min}$, and the cell pellets washed twice with PBST. The cells were then stained with $500 \mu \mathrm{L}$ PBS solution containing propidium iodide $(50 \mu \mathrm{g} / \mathrm{mL}), \mathrm{RNase} A(100 \mu \mathrm{g} / \mathrm{mL})$, and Triton X-100 $(0.2 \%)$, and incubated for 30 min at $4^{\circ} \mathrm{C}$. The cells $(20,000-30,000)$ were analyzed by flow cytometry. All the data were analyzed using the Cell Quest software. This procedure was repeated thrice.

\section{CCK-8 assay}

The cell number was determined by pre-incubating a cell suspension (100 $\mu \mathrm{L} /$ well) inoculated on a 96-well plate in a humidified incubator. The CCK-8 solution $(10 \mu \mathrm{L})$ was added to each well of the plate. The plate was incubated for $4 \mathrm{~h}$ in the incubator. The absorbance was 
measured at $450 \mathrm{~nm}$ using a microplate reader. The cell proliferation and cytotoxicity assays were performed. The cell suspension $(100 \mu \mathrm{L}$ each; 5000 cells/well) was dispensed on a 96-well plate. The other steps were similar to the experiment used to determine the cell number. This experiment was performed in triplicate.

\section{Western blotting}

Blank control cells, NC cells, and experimental transfected cells were homogenized and lysed in protein lysis buffer and proteinase inhibitor $(30 \mathrm{mM}$ Tris- $\mathrm{HCl}, \mathrm{pH} 7.5,150 \mathrm{mM} \mathrm{NaCl}$, $1 \mathrm{mM}$ phenylmethylsulfonyl fluoride, $1 \mathrm{mM} \mathrm{Na}_{3} \mathrm{VO}_{4}, 1 \%$ Nonidet $\mathrm{P}-40$, and $10 \%$ glycerol). The protein concentration in the cell lysates was quantified using a standard bicinchoninic acid assay kit (Beyotime, Haimen, China). The total protein $(40 \mu \mathrm{g})$ was separated on a $10 \%$ sodium dodecyl sulfate polyacrylamide gel, and electrophoretically transferred to polyvinylidene difluoride (PVDF) membranes. Strips of the membranes were blocked for $1 \mathrm{~h}$ at room temperature with the blocking buffer (1X TBS, $0.05 \%$ Tween-20 with 5\% non-fat dry milk), incubated overnight at $4{ }^{\circ} \mathrm{C}$ with anti-AR antibodies, anti-Bcl-2 antibodies, anti-CapG antibodies, anti-Cyclin D1 antibodies (1:1000; Epitomics, Burlingame, CA, USA) or anti-GAPDH antibodies (1:2000; Prosci, Poway, CA, USA). The membranes were subsequently incubated for $1 \mathrm{~h}$ at room temperature with horseradish peroxidase-conjugated anti-rabbit IgG (1:5000; Santa Cruz Biotechnology, Santa Cruz, CA, USA). The peroxidase activity on the PVDF membrane was visualized on an X-ray film by means of the electrochemiluminescence western blot detection system (Yuehua, Guangdong, China). Bands on the films were scanned and quantified by densitometric scanning of the blots, and normalized against GAPDH.

\section{Trans-well assay}

Matrigel was thawed and incubated in a $4^{\circ} \mathrm{C}$ refrigerator overnight. The Matrigel $(1 \mathrm{mg} / \mathrm{mL})$ was diluted and mixed in serum free-cold RPMI-1640. The diluted matreigel $(100 \mu \mathrm{L})$ was added to the upper chamber of a 24 -well trans-well chamber. The trans-well chamber was incubated at $37^{\circ} \mathrm{C}$ for at least 4 to $5 \mathrm{~h}$ to allow for gelling. The cells were harvested from tissue culture flasks using trypsin/EDTA, and washed thrice with RPMI1640 supplemented with $1 \%$ FBS. The cells were resuspended in media containing $1 \% \mathrm{FBS}$, at a density of $10^{6} \mathrm{cells} / \mathrm{mL}$. The gelled matrigel was gently washed with warmed serum-free culture media. The cell suspension (100 $\mu \mathrm{L})$ was added to the matrigel. The lower chamber of the trans-well was filled with $600 \mu \mathrm{L}$ RPMI1640 containing $5 \mu \mathrm{g} / \mathrm{mL}$ fibronectin, as an adhesive substrate. The cells were incubated at $37^{\circ} \mathrm{C}$ for 20 to $24 \mathrm{~h}$. The trans-well chambers were then removed from the 24-well plates and stained with Diff-Quick solution (IMEB, San Marcos, CA, USA). Non-invaded cells (cells remaining on the top of the trans-well chamber) were scraped off using a cotton swab. The invaded cells were counted under a light microscope.

\section{RESULTS}

\section{Real-time quantitative PCR}

The expression of the CapG, TMPRSS1, EGFR, ETS-1, ERBB2, AKT, Cyclin D1, P21, $B c l-2$, and Bak1 mRNA transcripts was significantly lower $(P<0.05)$, while that of the $/ G F-1$, FGF8, C-FLIP, E-Cadherin, P2, TRADD, and Cadherin-11 mRNA transcripts was significantly 
higher in the siRNA group than in the NC group $(\mathrm{P}<0.05)$. The expression of the $A R$, Maspin, and FOXO1 mRNA transcripts in the siRNA group was similar to that in the NC group. These results are demonstrated in Figure 2.

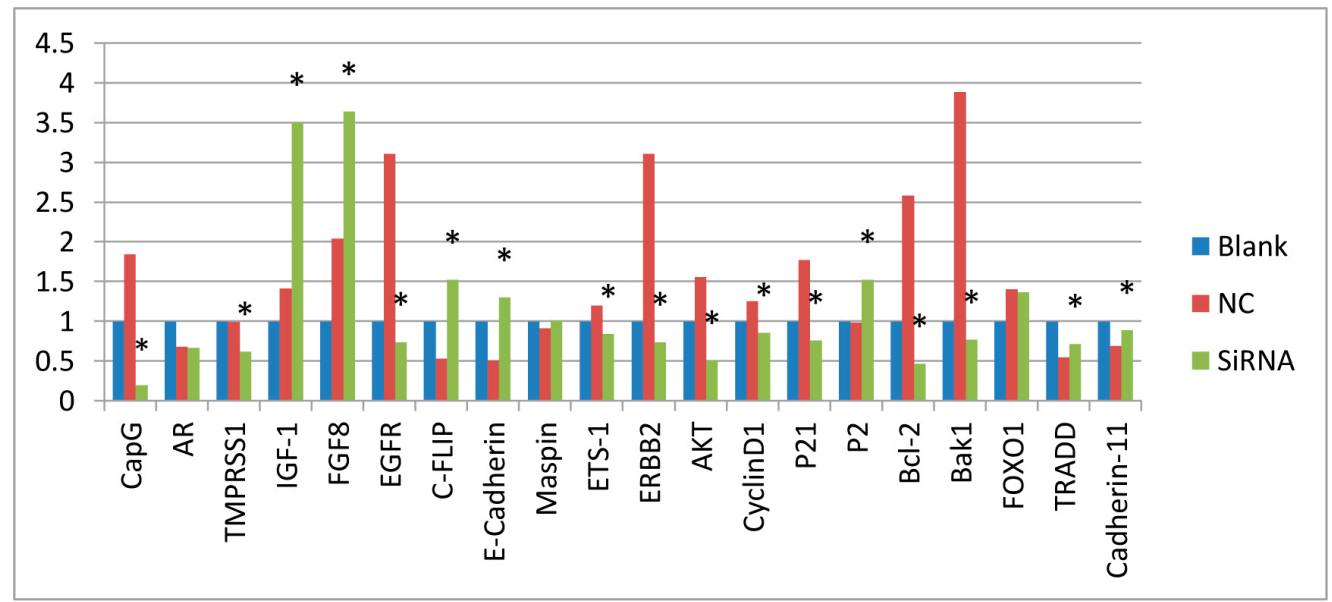

Figure 2. CapG, TMSSPP1, EGFR, ETS-1, ERBB2, AKT, Cyclin D1, P21, Bcl-2, and Bak1 mRNA transcripts are significantly lower in the siRNA groups compared to the negative control (NC) groups ( $\left.{ }^{*} P<0.05\right)$. The IGF-1, FGF8, C-FLIP, E-Cadherin, P2, TRADD, and Cadherin-11 mRNA transcript levels are significantly higher in the siRNA groups compared to the NC groups ( $\left.{ }^{*} P<0.05\right)$.

\section{Western blot analysis}

Figures 3 and 4 present the results of the western blot analysis. The levels of Bcl-2, Cyclin $D 1$, and CapG expression were significantly downregulated $(P<0.05)$ in the siRNA groups compared to the NC group. On the other hand, AR was similarly expressed in both the NC and siRNA groups.

\section{Cell NC siRNA}

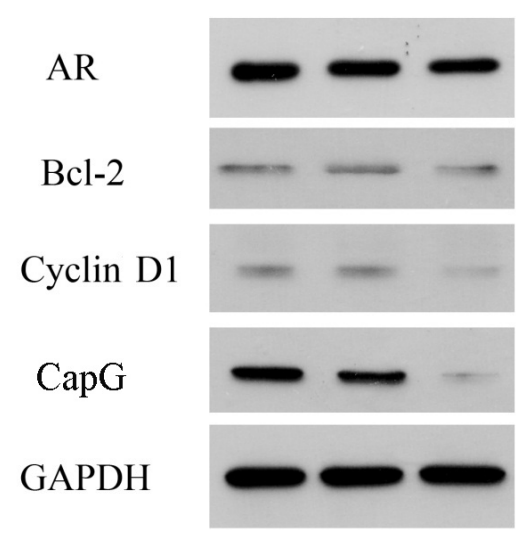

Figure 3. Results of the western blot analysis for AR, Bcl-2, Cyclin D1, and CapG in the three groups. NC = negative control. 


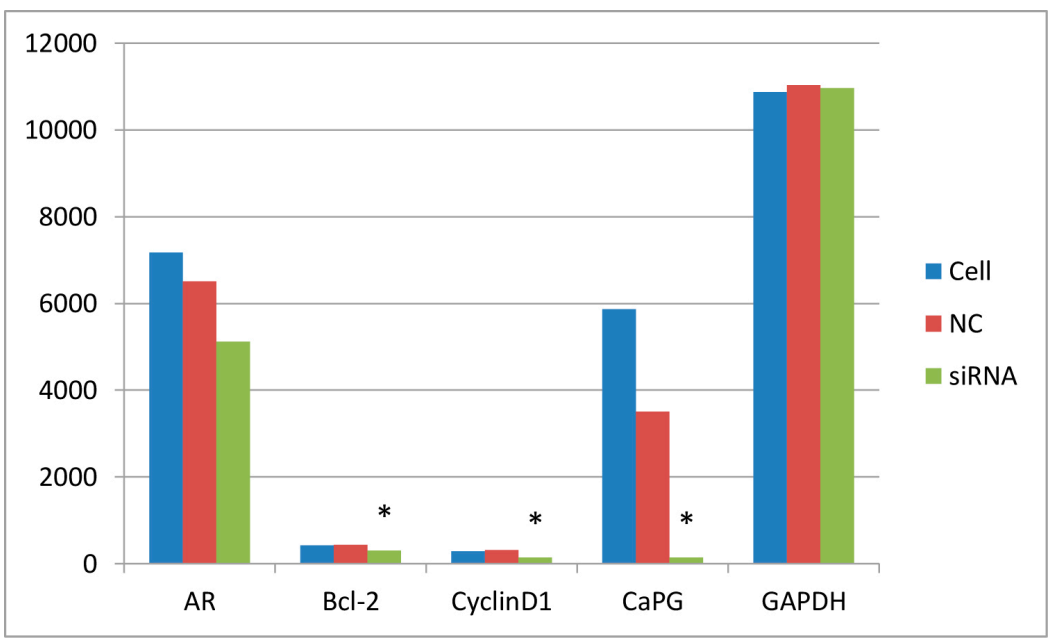

Figure 4. Bcl-2, Cyclin D1, and CapG expression is significantly downregulated ( $\left.{ }^{*} P<0.05\right)$ in the siRNA groups compared to the negative control (NC) groups.

\section{CCK8 assay}

In this assay, the effect of siRNA-mediated downregulation of CapG on DU145 cell growth was analyzed. The CCK8 assay was performed to investigate the viability of DU145 cells. The CapG siRNA-transfected DU145 cells showed a decrease in proliferation compared to the cells of the two control groups after $48 \mathrm{~h}$. The cell inhibition rate was $24.5 \%$ at $24 \mathrm{~h}, 35.4 \%$ at $48 \mathrm{~h}$, and $16.5 \%$ at $72 \mathrm{~h}$. The growth curve indicated that the cell proliferation was significantly slower in DU145 cells (transfected with CapG siRNA) compared to those transfected with siRNA-NC, or the untransfected cells $(P<0.05$; Figure 5$)$. We observed that the DU145 cell proliferation was restored with the decrease in the effect of CapG siRNA.

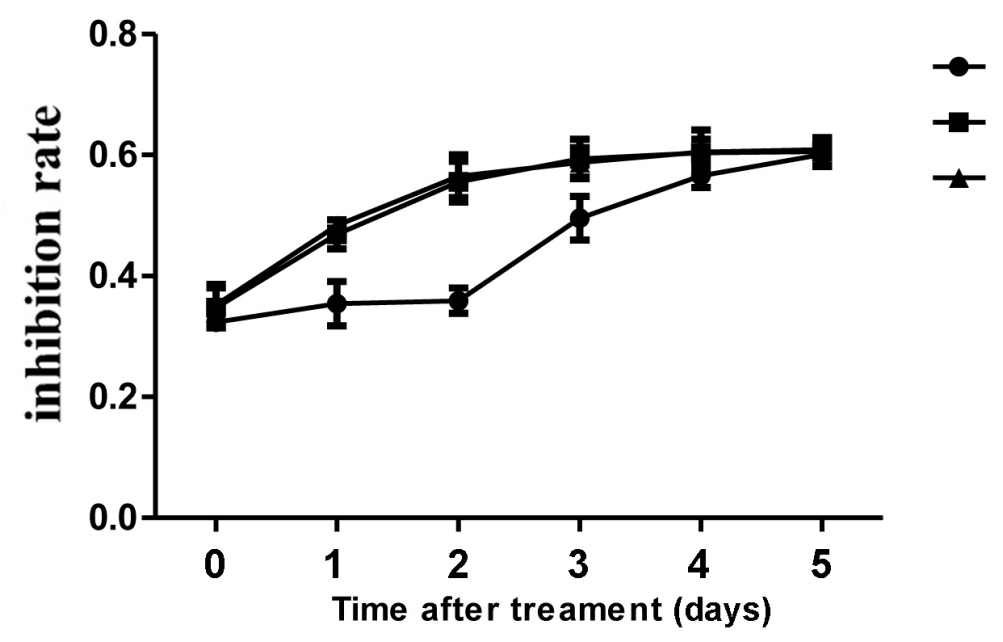

Figure 5. Status of DU145 cell proliferation determined using the CCK8 assay. 


\section{Trans-well migration assay}

CapG silencing could inhibit the migratory and invasive capacities of DU145 cells. The migratory capacity and invasiveness of DU145 cells was significantly decreased in the siRNA group compared to the NC cells $(\mathrm{P}<0.05$; Figure 6).

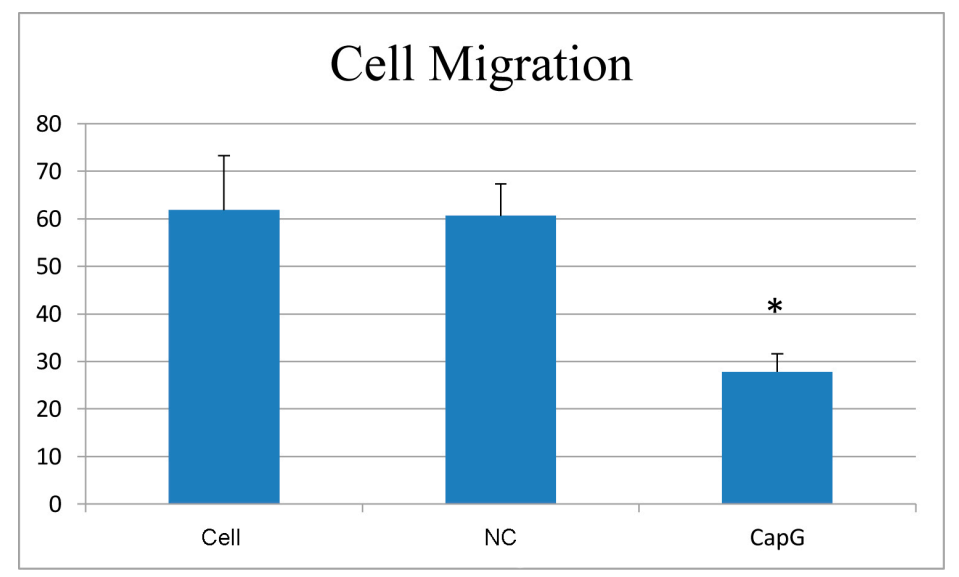

Figure 6. Migratory and invasive capacities of DU145 cells significantly decreased in the siRNA group ( $\left.{ }^{*} \mathrm{P}<0.05\right)$ compared to the negative control (NC) groups.

\section{DISCUSSION}

Prostate cancer is one of the most common cancers affecting elderly males; therefore, prostate cancer is an important cause of cancer-related deaths in Chinese men. The disease generally reaches an advanced state at the time of diagnosis (Pan et al., 2013). Therefore, prostate cancer is a prime candidate for further exploration of the CapG therapeutic approach. However, although many reports have focused on the CapG expression, few have discussed the expression of CapG in prostate cancer cells.

The function of CapG in the cytoplasm has been previously reported. Increased levels of the CapG protein have been reported in several tumors, including pancreatic ductal adenocarcinoma (lacobuzio-Donahue et al., 2003; Thompson et al., 2007) and glioblastomas (Lal et al., 1999). CapG caps, and therefore rapidly blocks, the growth of actin filaments, promoting the elongation of shorter actin filaments. It thus contributes to cell motility (Silacci et al., 2004) and membrane ruffling (Witke et al., 2001). Transient CapG knockdown experiments in the pancreatic cancer cell lines Suit-2, MiaPaca-2, and Panc-1 have resulted in a significant decrease in the wound healing capacity and cell motility (Thompson et al., 2007). Furthermore, van den Abbeele et al. (2007) have demonstrated that CapG is downregulated in the breast cancer cell line MDA-MB 231.

In this study, we have demonstrated that the suppression of the CapG gene expression in prostate cancer cell lines causes a significant decrease in its proliferative, invasive, and metastatic capacities. This is consistent with the results of a previous study, which reported an increase in tumor invasiveness following CapG overexpression in another malignancy. Interestingly, it has been reported that CapG requires the active nuclear import to promote tumor invasion (De Corte et 
al., 2004). The prevention of nuclear accumulation of CapG in the MDCK cells abolished collagen invasion; meanwhile, the restoration of nuclear import of CapG also restored collagen invasion in these cells. In order to identify possible cellular pathways with which CapG promotes proliferation and invasion, other cytokines related to the CapG were also detected. The $\mathrm{Bcl}-2$ gene is one of the most important apoptosis regulatory factors. Bcl-2 does not affect cell proliferation; however, it is known to suppress cell apoptosis, thereby extending the life span of the cell (Geng et al., 2013). The overexpression of cyclin D1, an important cell cycle regulatory factor, is a primary characteristic of various primary tumors (Kim et al., 2014; Ripple et al., 2014).

CapG interacts with microtubule-dependent organelles during the cell cycle (Hubert et al., 2009), possibly mediating cross-talk between the actin cytoskeleton and microtubule-based organelles involved in mitosis. CapG is imported into the nucleus by NTF2, Ran GTPase, and nucleoporin Nuc62 (van Impe et al., 2008), which was observed in HEK293T, HeLa, and MDCKAZ cells. In this study, the suppression of CapG was found to cause different effects on the other oncogenes in prostate cancer. This suggests that an excess of CapG may not be sufficient to alter the cell cycle dynamics, indicating a combined effect with other oncogenes. However, this does not necessarily exclude the role of CapG in the cell cycle processes of prostate cancer.

In summary, the suppression of CapG gene expression in a prostate cancer cell line was shown to cause a significant decrease in the proliferative, invasive, and metastatic abilities of prostrate cancer cells; however, CapG overexpression does not individually alter the cell cycle dynamics, requiring assistance from other oncogenes. The embedded mechanism of the interaction of CapG with other oncogenes, thereby influencing the cell cycle, remains to be further analyzed.

\section{Conflicts of interest}

The authors declare no conflict of interest.

\section{ACKNOWLEDGMENTS}

Research supported by the Natural Science Foundation of Guangdong Province (\#S2012010008156 and \#S2013040016823).

\section{REFERENCES}

De Corte V, Van Impe K, Bruyneel E and Boucherie C (2004). Increased importin-beta-dependent nuclear import of the actin modulating protein CapG promotes cell invasion. J. Cell Sci. 117: 5283-5292.

Geng ZM, Zhang M, Pan XT and Wang L (2013). Bcl-2 gene silencing by RNA interference inhibits the growth of the human gallbladder carcinoma cell line GBC-SD in vitro and in vivo. Oncol. Rep. 30: 793-800.

Hubert T, Van Impe K, Vandekerckhove J and Gettemans J (2009). The actin-capping protein CapG localizes to microtubuledependent organelles during the cell cycle. Biochem. Biophys. Res. Commun. 380: 166-170.

lacobuzio-Donahue CA, Maitra A, Olsen M, Lowe AW, et al. (2003). Exploration of global gene expression patterns in pancreatic adenocarcinoma using cDNA microarrays. Am. J. Pathol. 162: 1151-1162.

Karantanos T, Corn PG and Thompson TC (2013). Prostate cancer progression after androgen deprivation therapy: mechanisms of castrate resistance and novel therapeutic approaches. Oncogene 32: 5501-5511.

Kim MH, Ham O, Lee SY and Choi E (2014). MicroRNA-365 inhibits the proliferation of vascular smooth muscle cells by targeting cyclin D1. J. Cell Biochem. 115: 1752-1761.

Kimura K, Ojima H, Kubota D, Sakumoto M, et al. (2013). Proteomic identification of the macrophage-capping protein as a protein contributing to the malignant features of hepatocellular carcinoma. J. Proteomics 78: 362-373.

Lal A, Lash AE, Altschul SF, Velculescu V, et al. (1999). A public database for gene expression in human cancers. Cancer Res. 59: 5403-5407. 
Morofuji N, Ojima H, Onaya H, Okusaka T, et al. (2012). Macrophage-capping protein as a tissue biomarker for prediction of response to gemcitabine treatment and prognosis in cholangiocarcinoma. J. Proteomics 75: 1577-1589.

Pan B, Zheng S, Liu C and Xu Y (2013). Suppression of IGHG1 gene expression by siRNA leads to growth inhibition and apoptosis induction in human prostate cancer cell. Mol. Biol. Rep. 40: 27-33.

Partheen K, Levan K, Osterberg L, Claesson I, et al. (2008). Four potential biomarkers as prognostic factors in stage III serous ovarian adenocarcinomas. Int. J. Cancer 123: 2130-2137.

Ripple MJ, Parker Struckhoff A, Trillo-Tinoco J, Li L, et al. (2014). Activation of C-Myc and Cyclin D1 by JCV T-Antigen and ß-catenin in colon cancer. PLoS One 9: e106257.

Rosenfeld MG, Lunyak VV and Glass CK (2006). Sensors and signals: a coactivator/corepressor/epigenetic code for integrating signal-dependent programs of transcriptional response. Genes Dev. 20: 1405-1428.

Shao F, Zhang R, Don L and Ying K (2011). Overexpression of gelsolin-like actin-capping protein is associated with progression of lung adenocarcinoma. Tohoku J. Exp. Med. 225: 95-101.

Siegel R, Naishadham D and Jemal A (2013). Cancer statistics, 2013. CA. Cancer J. Clin. 63: 11-30.

Silacci P, Mazzolai L, Gauci C, Stergiopulos N, et al. (2004). Gelsolin superfamily proteins: key regulators of cellular functions. Cell Mol. Life Sci. 61: 2614-2623.

Thompson CC, Ashcroft FJ, Patel S, Saraga G, et al. (2007). Pancreatic cancer cells overexpress gelsolin family-capping proteins, which contribute to their cell motility. Gut 56: 95-106.

van den Abbeele A, de Corte V, van Impe K, Bruyneel E, et al. (2007). Downregulation of gelsolin family proteins counteracts cancer cell invasion in vitro. Cancer Lett. 255: 57-70.

van Impe K, Hubert T, de Corte V, Vanloo B, et al. (2008). A new role for nuclear transport factor 2 and Ran: nuclear import of CapG. Traffic 9: 695-707.

Witke W, Li W, Kwiatkowski DJ and Southwick FS (2001). Comparisons of CapG and gelsolin-null macrophages: demonstration of a unique role for CapG in receptor-mediated ruffling, phagocytosis, and vesicle rocketing. J. Cell Biol. 154: 775-784.

Wu JH, Tian XY and Hao CY (2011). The significance of a group of molecular markers and clinicopathological factors in identifying colorectal liver metastasis. Hepatogastroenterology 58: 1182-1188.

Zheng Z, Li J, He X, Chen X, et al. (2010). Involvement of RhoGDI2 in the resistance of colon cancer cells to 5 -fluorouracil. Hepatogastroenterology 57: 1106-1112.

Zhu WY, Hunag YY, Liu XG, He JY, et al. (2012). Prognostic evaluation of CapG, gelsolin, P-gp, GSTP1, and Topo-Il proteins in non-small cell lung cancer. Anat. Rec. 295: 208-214. 